\title{
Brain circulation: Evolution and development with related clinical implication, with particular attention to hippocampus evolution
}

\author{
Payam S. Pahlavan \\ Department of Pathology, Dr. Everett Chalmers Regional Hospital, Fredericton, \\ New Brunswick, E3B 5N5, Canada
}

\begin{abstract}
Reviewing the brain circulatory system evolution and development is crucial as it helps understand brain circulation diseases including stroke. Literature review shows limited research on this topic. We tried to collect all available sources and present a summarized, compact evolutionary data to help further investigation and research on this important subject.
\end{abstract}

Keywords: brain circulation, evolution, development, variation, duplication

\begin{abstract}
Abbreviations
central nervous system (CNS), neurovascular unit (NVU), ICA: internal carotid artery, ACA: anterior cerebral artery, MCA: middle cerebral artery, PCOM: posterior communicating artery, ACOM: anterior communicating artery, PCA: posterior cerebral artery, PCOM: posterior communicating artery, PICA: posterior inferior cerebellar artery, VA: vertebral artery, AICA: anterior inferior cerebellar artery, RAH: recurrent artery of Heubner, AChA: anterior choroidal artery, PTA: persistent trigeminal artery, SCA: superior cerebellar artery, ITDPCS; infarct in the territory of the deep perforators from the carotid system, PI: pulsatility index, WMH: white-matter hyperintensity, NF: nasal fossa, PAA: pro-atlantal artery.
\end{abstract}

\section{INTRODUCTION}

From a gross anatomical level, the pathways of blood vessels and nerves appear in a good harmony. From a cellular level, proliferating vessels and neurons get benefit from similar specialized structures that operate target tissue pathway through sensing their local environment (1). The vascular and nervous systems have developed a complex relationship within the central nervous system (CNS) itself. This connection is found at the surface between the vascular and nervous systems that is generally recognized as the neurovascular unit (NVU). The NVU is composed of endothelial cells, neuroglial cells, pericytes, and neurons which are firmly coupled to control cerebrovascular function (2). Most scientists believe that the modern nervous system evolved prior to the circulatoryr system. It is believed that the evolution of brain circulation provides evidence to progressive recruitment of already existing vascular networks to supply newly developed cortical territories, rather than development of arterial networks by itself.

After closure of the neural tube (around week 4), it is encircled by a dense connective tissue, the primitive meninx (weeks 5-8). This primitive meninx which is a primitive network of mesenchymal cells, contains primitive vascular loops that are developed by vasculogenesis from the primitive dorsal aorta and cardinal veins, and through them, they are connected with the primordial vascular organ that is initially developed over the yolk sac. The meninx follows the cortical folds that appear during development of the brain. It fills the interhemispheric fissure which forms between the two lateral 
telencephalic bulges and eventually will become the falx cerebri. At this early stage (when the embryo $<4 \mathrm{~mm}$ length), there is no differentiation into arteries and veins. The irregular network of endothelial vascular channels constitutes a early developed of germinal bed of endothelium rather than a true circulatory system. The primitive meninx differentiates into three separate layers, which will ultimately become the dura mater, the arachnoid, and the pia mater (3). Eventually, the angioblastic cells from the meninx become applied to the superficial surface of the developing brain, then penetrate the surface and extend perpendicularly to the pial surface between the glial elements. This so-called endothelial "buds", forms an extensive capillary-like network of endothelial cells (4).

As the cephalic portion of the neural tube grows and expands to form the three primary brain vesicles (rhombencephalic, mesencephalic, and prosencephalic vesicles), the primitive meninx evolves further to better supply the neural tissue through invagination into the roofs of the prosencephalic and rhombencephalic vesicles. This process forms the primordia of the choroid plexuses (approximately between weeks 5-7). At this stage, diffusion of nutrients to the neural tissue is both peripheral from the primitive meninx and through ventricular from the developing choroid plexuses (5). From the point of morphogenesis of the cerebral vasculature, this complex differentiation is crucial: it leads to the early differentiation of specific choroid feeders within the meningeal vascular meshwork from which all brain arteries eventually evolve (6).

One of the earliest researches in understanding cerebral vascular development comes from Streeter studies (7). He described five periods in the development of the cerebral vasculature. The first period is the appearance of primordial endothelium-lined channels that create a primitive plexus within the head. There is no circulation in the vessels at this early stage. This stage matches the third week of fetal development in human fetus. The second period is when the primitive plexus begins to organize into arteries, veins and capillaries. Connection of this system with the primitive aortic vessels initiates the circulation in the head which coincides with the late fourth week of fetal development. During the third period, there is a division of the vasculature of the head into three separate systems involving the external structures, the dura, and the cerebrum which matches the weeks $6^{\text {th }}$ and $7^{\text {th }}$ of fetal age. During the fourth period, the vasculature develops and matures in response to the growth of the brain. This period matches with the $9^{\text {th }}$ week of the fetal age. Fifth period includes histologic changes in the wall of the vessel that result in the appearance of mature arteries, capillaries, and veins. This period extends beyond birth (8).

\section{Evolution of the circulatory system of the central nervous system in different species}

In fishes, there is primitive cerebellum, also called a small olfactory lobe, and a primitive cortex, composed of three layers. The higher center of movement is controlled by what will eventually become the basal ganglia in higher developed species. The spinal cord will basically remain unchanged. The spinal cord is supplied by the segmental radiculomedullary arteries at the thoracic segment and by segmental arteries arising from the vertebral arteries (VA) at the cervical segment. The VAs are the result of longitudinal anastomosis between segmental arteries. The arrangement in the brain is similar. It presents by two ascending large longitudinal vessels in the neck that will enter the cranial cavity through the same way of segmental radiculomedullary vessels in the spinal cord. Following entering the cranial cavity, the large longitudinal vessel (carotid) gives rise to internal carotid artery (ICA) which gives rise to paired longitudinal arteries which run the length of the brain. The artery branch that projects forward is called the "cranial ramus" and the artery branch that projects caudally is the "caudal ramus". The "cranial ramus" is the predecessor of the anterior cerebral artery (ACA) and middle cerebral artery (MCA). The caudal ramus would become the predecessor of the posterior communicating artery (PCOM) and parts of the basilar artery. The basilar artery has already started fusing at midline at this level of development (9).

At this level, no significant functional anastomosis exists between the "caudal ramus" and vertebral artery system of the spinal cord. Brain, cerebellum and prosencephalon are supplied by ICA. Then, through the evolutionary process, the "cranial ramus" makes two branches, the "medial olfactory artery" which gives rise to the ACA and the "lateral olfactory artery". The "caudal ramus" also gives 
rise to tectal artery which feeds the posterior superior of the brainstem. In addition, many small perforators come off all these vessels. Small segmental anastomosis between the carotid and vertebral artery gradually starts (9).

In amphibians, a primitive hippocampus and a primitive basal ganglia start to form. The cerebellum also enlarges in size compared to fishes. The lateral olfactory artery divides to two main branches, the lateral striate artery and the posterior telencephalic artery. On the caudal ramus, the tectal-cerebellar complex starts to come to exist and will eventually supply a developing choroid plexus through a posterior choroidal artery branch. In amphibians, no effective anastomosis between carotid and vertebral system exists (9).

In reptiles, from the lateral striate artery of the rostral division, multiple perforator vessels arise that will eventually supply the developing hemispheres. These perforator vessels will eventually become the MCA. A fused midline olfactory artery in some reptiles is observed. The posterior telencephalic artery is enlarged and supplies the posterior portions of the cerebral hemispheres. The posterior cerebral artery (PCA) does not exist in reptiles and its predecessor (the tectal artery) supplies a smaller territory in the brain circulation. An enlarging cerebellum and its caudal vermian portion are supplied by the homolog of the posterior inferior cerebellar artery (PICA) as part of the distal basilar system (9).

In birds, developmentally a distinct MCA, as a prominent perforator emerges from lateral striate vessels, driven by rapidly enlarging cerebral cortical size. The perforator vessels arise from all sections of the circle of Willis and MCA. The ACA segment dominant perforator which is the analogous to recurrent artery of Heubner (RAH) is present. In birds, PCA continues as the dominant source to supply parieto-occipital area. The newly formed tectal artery extpands its coverage and forms an anastomosis with the anterior choroidal system. PICA continues to be supplied by the anterior circulation via cranio-petal flow into the basilar artery. The vertebral system is confined to the spinal cord or in some cases with the lower medulla-vermian region (9).

In mammals, with the development of the caudal ramus with its enlarging cerebellum and posterior telencephalon, there is an increasing demand on the carotid system. In mammals as advanced as the sheep, the vertebral system does not contribute to the brain circulation. However, in higher mammals the carotid system progressively reaches to its higher functional limit and brainstem and cerebellar territory will be supplied by the vertebro-basilar system. At first, this starts to the PICA region. Then evolutionary in higher mammals, the VA adds portions of basilar territory that will result in reversal of cranio-petal basilar flow to the cranio-fugal form which is observed particularly in monkeys and apes (9).

\section{Evolution, embryology and anatomical level in human brain circulation}

At early embryonic developmental stage, the vessels are grouped in plexi. These vessels are initially paired and encircle the CNS. Starting from the basic level, the neural tube can be considered and imagined as a cylindrical mass. For a short period of time after closure of the neural tube, nutritional support can adequately be provided by simple diffusion. When limits of diffusion are exceeded and demand increased, dedicated vascular supply to the neural tube and adjacent tissues is going to be established via segmental (metameric) vessels arising from the dorsal aorta. Each segmental vessel supports its endodermal, ectodermal, and mesodermal tissue. Following these evolutional steps, longitudinal anastomoses are established between segmental vessels, thereby giving rise to new vessels extending from cranial to caudal along the length of the cord.

As these longitudinal vessels develop, hemodynamically a need for having a segmental radiculomedullary vessel at each level is decreased. Therefore, later on, most segmental vessels would supply only a region of a segmental nerve root. In the next step in the process of circulation evolution, the cord is then supplied by the "anterior spinal" artery ventrally. Then, a loose network of arteries with two dominant channels would shape the "posterior spinal" arteries. As the cord continues to grow, perforator or sulco-comissural branches would come into existence.

If primitively and basically consider that a developing brain is a progressively enlarging segment of the cord, it requires a larger surface and deep 
perforator vessels to essentially understand the evolution and vascular variation in the brain circulation. It is believed that the arrangement in the brain is similar to cord circulation evolution. The first six pairs of aortic arches and their intersegmental arteries compose the vascular formation which eventually will develop into adult cerebral circulation. The first pair of aortic arches gives rise to the primary head plexus. From this plexus, the external and internal carotid arteries will arise. The ventral roots between the third and fourth arches form the common carotid artery and the dorsal roots regress. Two large longitudinal vessels, here they are the carotids, ascend the neck and enter the cranial cavity. This ascending and devotement is in a way similar to that of segmental radiculomedullary vessels in the cord. Inside the cranium, the internal carotid artery (ICA) would give rise to paired longitudinal arteries which run the total length of the brain (10). During evolution, the ICA projects to its final placement with the first cervical position starting above the carotid bulb and collateral branches that originates between the segments. The terminal segments of the ICAs divide in plexiform channels toward the Ratke's pouch to communicate with the opposite side and in the primitive trigeminal artery, extending dorsally to communicate with the bilateral longitudinal neural arteries on the hindbrain wall (11). Eventually, most of these vascular communications regress, with the fused portion of the longitudinal neural arteries forming the midline basilar artery, and the posterior communicating artery replacing the tentorial artery as the source of collateral supply between the anterior and posterior circulation.

Each ICA grows rostrally and at the level of the optic vesicle, divides into cranial and caudal branches. The cranial branch will give rise to anterior choroidal artery (AChA), MCA and primitive olfactory artery and also the anterior cerebral artery (ACA) at its end. The caudal branch gives rise the diencephalic, mesencephalic and posterior choroidal branches (12). The ICA first gives rise to primitive olfactory artery (POA), which eventually terminates in the nasal fossa (NF). The secondary branch of the POA is the medial olfactory artery (MOA), which constitutes future ACA and the lateral olfactory artery.

The segmental anastomosis which already exists between the carotid and vertebral systems would start to regress as the vertebrobasilar system develops. Next, in the evolutionary process of CNS circulation, the lateral striate (the precursor of Heubner) branch of the rostral division gives rise to multiple "perforator" vessels that will supply the developing hemisphere. These "perforator" vessels will eventually become the MCA. In fact, the MCA is phylogenetically a relatively late acquisition hypertrophied perforator branch of the ACA. In evolutionary process, the MCA gives origin to two main groups of perforators: the medial and lateral lenticulostriate arteries. The ACA also gives rise to the anterior lenticulostriate and the recurrent artery of Heubner (RAH) (13).

The lenticulostriate arteries are terminal vessels that have no anastomosis and will supply future basal ganglia in the brain circulatory system. In the early embryonic stages, the germinal matrix forms the entire wall of the cerebral vesicles. This layer is fed by a dense rete of thin- walled vessels that is connected to the surface of the vesicles by large connections. Due to migration and development of cerebral cortex and white matter, the germinal layer and its vascular rete will become more deeply located in the cerebral hemispheres. The rete remains connected to the surface by ventriculopetal parallel running channels. From the third fetal month, the germinal layer and its rete would start to regress. This occurs first around the third and fourth ventricles and later around the posterior parts of the lateral ventricles. Only from the fourth fetal month the deep perforating vessels would start to differentiate in arteries with specific cortical vessels. Further regressing of capillary rete until birth leaves the ventriculopetal (medullary) branches in the white matter around the frontal horn and the body of lateral ventricle that would end with some distance from the ventricular wall, opposite to the ventriculofugal branches developed from deep perforating branches of the basal ganglia (lateral striatal branches) (11).

Further during evolution, both olfactory arteries fuse in the midline and make the new anterior communicating artery (ACOM). Physiologically, the circle of Willis, unites the ICA and vertebrobasilar systems and is formed by anastomosis among the ICA, pre-communicating part (A1) of ACA, ACOM, pre-communicating part $(\mathrm{P} 1)$ of posterior cerebral artery (PCA) and posterior communicating artery (PCOM) (14). 
Reviewing variations in the circle of Willis revealed that most of the branches are derived from the ICA, gradually from the third arch artery (15). At the 5 to $7-\mathrm{mm}$ fetal stage, the caudal branch of the ICA makes anastomosis with the ipsilateral longitudinal neural artery. With blood flow via this anastomosis as well as via the pro-atlantal intersegmental artery $(=\mathrm{a}$ branch arising from dorsal aorta), the primitive arteries of otic, hypoglossal and trigeminal arteries which already given rise from the ICA will start to regress (12).

The perforator vessels arise from all portions of the circle of Willis, and mainly from the MCA. The phylogenetically novel contribution of vertebral system in brain circulation is optimized in the human, where the PCA, is functionally acquired from the posterior circulation, even though it developmentally belongs to the carotid arteries (9). The connection and joining of the caudal branch of the ICA and the longitudinal neuronal artery makes the PCA (12).

On the other hand, the posterior inferior cerebellar artery (PICA) directly derives from basilar artery. Superior cerebellar duplications are common, but transfer of overall territory to another cerebellar branch is uncommon. As a matter of fact, the development of the cerebellar arteries occurs later in brain circulatory development, which totally matches the delayed development of the cerebellum compared to the cerebrum (16). Then PCOMs start to form and continues to supply the PCA through carotid arteries. As mentioned earlier, the MCA arises as a dominant vessel from joining few lateral striate perforators of the ACA. If a MCA duplication occurs (in $0.2 \%$ to $2.9 \%$ of cases) which originates from another dominant striate perforator, an accessory MCA would come to existence. Manelfe classifies the accessory MCA into three types: type 1 is an uncommon vessel that arises from the ICA at a point proximal to its bifurcation, type 2 is another variation that originates from the proximal portion of the ACA, and type 3 is the vessel that originates from the distal portion of the A1 segment of the ACA proximal to the ACOM (17). The end arteries that later originate perpendicularly from the major vessels including the MCA, lack collaterals from nearby arteries which make them more susceptible to arterial hypertension. These arteries will supply the vascular centrencephalon which includes phy- logenetically the older sections of the brain (18). Continuing branching of a separate striate perforator gives rise to RAH (medial striate artery) which is in fact a medial ACA perforator (9). AChA originates from the ICA or occasionally from the MCA and has a rich vascular territory, making two branches, anterior part supplying different anatomical structures including anterior part of optic tract and internal part of globus pallidus and the posterior part supplying structures like the uncus and piriform cortex (19). AChA supplies a significant portion of cortical territory that is also incorporated by the PCA. This supply continues nearly toward late stage in the evolutionary process. By 7- to $12-\mathrm{mm}$ fetal stage, the longitudinal neural arteries would join to form the basilar artery. The intersegmental arteries that arise from the posterior aorta to supply the cervical somites would connect at their dorsal ends in a rostro-caudal fashion.

Following this evolutionary process, all communications to each dorsal aorta regress except that from the sixth intersegmental artery. This dorsal channel becomes the vertebral artery and the sixth intersegmental artery contributes to the subclavian artery (12). From the basilar artery the superior and anterior inferior arteries start to appear and from the vertebral arteries, the posterior inferior cerebellar arteries form. Occasionally, following completion of development, the level of inter communication is incomplete and large portions of the cortex would remain under AChA control and as a result the PCA contribution is reduced. This is sometimes called as duplicated PCA. Persistent trigeminal artery (PTA) is the most common embryonic carotid-vertebrobasilar anastomosis at the mid-basilar level that constitutes persistence of segmental ICA-basilar connection along the trigeminal nerve. Distal to the origin of PTA, the hypoglossal artery enters the cranium through the hypoglossal canal and makes the anastomosis between the distal vertebral and the proximal ICAs. The direct perforators from the ICA supply an area at the junction of the anterior part and the genu of the internal capsule and the anterior poles of the putamen and pallidum (20).

Pro-atlantal artery is an embryonic carotid-vertebral anastomosis. Two types of pro- atlantal arteries are well recognized, both of them arise from the carotid artery and then enter the cranium through foramen magnum. The type 1 pro-atlantal artery 
(also called pro-atlantal intersegmental artery) arises from the caudal ICA. It ascends to the level of the occipito-atlantal space without passing through the transverse foramen of any cervical vertebra. This artery takes a dorsal course cephalad to the transverse process of $\mathrm{C} 1$ and then travels rostrally to enter the foramen magnum. The type 2 pro-atlantal artery (also called $\mathrm{C} 1$ intersegmental artery) arises laterally from the external carotid artery and remains more exterior than the type 1 artery. It joins the course of the horizontal portion of the VA before entering the foramen magnum (21).

Important to mention that the pro-atlantal artery enters the skull through the foramen magnum and the hypoglossal artery enters through the hypoglossal canal (22).

Following development of the anterior circulation, the basilar artery is shaped through joining of multiple vessels belonging to the longitudinal neural system,. This process constitutes of several stages that could make multiple basilar artery variations, which can be considered from the standpoint of "extent of fusion" and "completeness of fusion" (i.e. fenestrations). Variations of basilar artery could include: 1) "Short basilar artery" that is equivalent to relative lack of fusion of the caudal segment, 2) remains unjointed at the top that is equivalent to "unzipping" at the basilar tip, 3) fenestrated which constitutes a broken fusing in the middle (9). An example of variation in basilar artery is when the top of the basilar artery is separated in two, so that one or both superior cerebellar arteries (SCA) originate from the P1 segment. Fenestration of basilar artery is common but has no clinical significance, except in a situation when it is too short and mimics a dissection.

\section{Duplications and variations}

Some duplications or variations in the brain circularly system described earlier. In general, duplication in arteries during brain circulation development is occasionally seen and the most commonly duplicated vessel is the SCA.

In reviewing the anterior circulation variations, the majority of anatomic variations have been reported in the ACOM section, showing multiple anatomic complexity and diversity. The ACOM, A1 and A2 segments of ACAs and the RAH combined with their perforators and other branches are often referred as the ACOM complex. Variations can be described in terms of two factors; fusion and pattern of branch takeoff. The spectrum of variations could extend from non-fusion (i.e., there would be no ACOM, multiple types of short segment fusions (duplicated ACOMs) or elongated segments merging (unpaired or azygous arrangements) (23). The unpaired type is the one where a long segment of merging divides into two branches, each of which gives rise to individual cortical branches to its specific hemisphere. The Azygous type (Azygos ACA) is a rare variant where the ACA is merged through its entire length and the individual branches to each hemisphere arises from a common trunk. In other words, the Azygos ACA involves a common trunk in the $\mathrm{A} 2$ segment above the ACOM. Its prevalence is between $0.3-2 \%$ (24).

Regarding branch takeoff variations, any takeoff variation could be possible. One of them that is clinically significant is the arrangement at the ACOM region. The fronto-polar branch may arise from the ACOM region. In addition, the separation of calossomarginal-pericalossal artery may occur anywhere along the length of ACA, starting from ACOM to the genu of the corpus callosum. If this separation occurs at the ACOM region, a threebranched appearance could result with pericalossal and calossomarginal arteries arising from one side, and A2 branch from the other side (9).

Between rare variations, the thalamotuberal artery may arise from the MCA but more commonly it originates from the PCOM (13).

\section{Hippocampus}

The hippocampus and fornix have long been considered part of the anatomic rhinencephalon. Experiments have demonstrated that these structures are without olfactory function (1). In different species that have been studied, including fish, amphibians, reptiles and mammals, relational representations are moderated by structures in the brain that phylogenetically derive from the inner medial part of the telencephalic anlage (2). In mammalians, this section is named the hippocampal formation. It is suggested that there is a correlation between the volume of the hippocampal formation and level of spatial performance in different species. The mammalian hippocampal formation represents one of the phylogenetically oldest cortical areas (3). 
The hippocampus (medial pallium) is a part of the cerebral cortex that evolutionally originates from the medial edge of telencephalic pallium. The medial pallium that is present in all vertebrates is phylogenetically characterized by features like connections to other brain regions. Also contains areas that are more specialized and varies in vertebrate groups like difference in cyto-architectural organization (4).

Nonmammalian vertebrates do not contain a similar dentate gyrus structure as mammals have and, hippocampal neurogenesis in them appears to be similar to the neurogenesis elsewhere in the brain (5).

In reptiles, the medial pallium (limbic) is a three-layered cortex, that contains medial (MC) and dorsomedial (DMC) subdivisions. The reptilian $\mathrm{MC}$ and $\mathrm{DMC}$ are homologous to the dentate gyrus and Ammon's horn of the mammalian HF, respectively. In addition, the adjacent medial portion of dorsal cortex (DC) is phylogenetically similar to the transitional entorhinal cortex of the mammalian hippocampus. Pyramidal-like neurons that are present in the reptilian medial pallium appear to be an apomorphic feature of amniotes. The MC of reptiles receives afferent projections from several telencephalic cortical regions. The DMC is mainly connected with the MC. In addition to reciprocal connections with $\mathrm{MC}$, there is a significant commissural connection between the DMCs of the two hemispheres. Both MC and DMC are reciprocally connected with the septum and would receive ascending connections from the anterior dorsomedial nucleus of the dorsal thalamus, the mammillary body, periventricular areas of the hypothalamus, the raphe nuclei, the locus ceruleus and also the reticular formation (4).

Earlier it was believed that the medial pallium and dorsomedial pallium that together is called as hippocampal formation (HF) was the homologue of the mammalian hippocampus. Both areas develop from the same region of the enlarging prosencephalon and both show a similar developmental gene expression pattern. In birds, the cortex of the medial surface of the pallium joins with more ventrally located pallial structures. As a result, the typical layered structure of the cortex would no longer be seen (3). The avian HF has a layered pattern mostly seen close to the "V" shaped cell layers in medial
HF. Bitufted spinous neurons, which resemble mammalian hippocampus pyramidal cells, populate the "V" layer. Also, multipolar spinous neurons populate more dorsomedial regions. Shared extra-HF/hippocampal connectivity includes similarly organized connections with the septum, lateral hypothalamus, amygdala (phylogenically the archipallium in birds), brainstem monaminergic nuclei and telencephalic sensory processing regions. The avian HF and mammalian hippocampus also share a generally similar neurochemical profile. At the electrophysiological level, both the mammalian hippocampus and avian HF display NMDA dependent and NMDA-independent changes in synaptic efficacy referred to as long-term potentiation. The evolution of hippocampal/HF organization may be characterized by inertia with respect to the combination of spatial dimensions that can determine variation in a neuron's action potential firing rate, but with distinguished plasticity in the way neurons with different spatial response properties are assembled into functional organizations (4).

Mammalian adult hippocampal formation and neurogenesis is characterized by evolutionary forces that are involved in the benefit of natural selection that are connected with the mammalian dentate gyrus. The mammalian hippocampus is generally described as a three layered structure containing three major subdivisions: the dentate gyrus, the Ammon's horn (CA1, CA2 and CA3) and the subiculum. In mammals, adult hippocampal neurogenesis starts from an ectopic precursor cell population that locates in a well-defined stem-cell center derived from the ventricular wall (6).

Comparative neuroanatomical and embryological studies have shown that in mammals the hippocampal formation most likely is homologous to parts of the medial cortex in reptiles. It is believed that the hippocampal formation derives from a dorsomedially located anlage in the newly developed brain which presents an overall likeliness with the dorsomedial cortex in reptiles (7). The hippocampus of rodents locates inside the telencephalon's caudal pole, whereas the primate hippocampus resides deep within the medial temporal lobe. However, the mammalian hippocampus always develops in the telencephalon's dorsomedial sector and remains at that location in the most primitive mammalian lineages including monotremes and marsu- 
pials (8). Therefore, one can predict that the hippocampal homolog in non-mammals should likewise occupy a dorsomedial position, at least during the early stages of development.

\section{Blood supply to hippocampus and its evolution}

Hippocampal arteries can be subdivided to superficial (leptomeningeal) and intra- hippocampal (deep) vessels.

\section{Superficial hippocampal arteries}

The hippocampal arteries mainly arise from the posterior cerebral artery (PCA) and to some extent from the anterior choroidal artery. The arterial supply of hippocampus that usually arise from the PCA include: anterior hippocampal artery that mostly gives rise from the PCA and less commonly from the anterior choroidal artery. Larger middle hippocampal artery most commonly gives rise from the PCA and posterior hippocampal artery mostly arises from the splenial artery or less likely from the PCA.

\section{Phylogeny and evolution of posterior cerebral artery}

The PCA is an advanced vessel, primarily made in the lower species prior to development of the MCA. The areas that it supplies include occipital and mesial temporal lobes (next to the tectum) which are phylogenetically more established than the bulk of frontoparieral areas which lately supplies by the MCA. The PCA originally belongs to the anterior, carotid circulation that arises as the carotid ends into the cranial and caudal rami. Transfer of the PCA territory to the vertebrobasular circulation is a procedure that appears to be necessary to make the phylognenetic start-point by the relatively large volume of brain that is supplied by the carotid system in higher species including the human. In many mammals the vertebrobasilar system does not primarily involve in the PCA supply and confines to the brainstem and cerebellum. This is the simple phylogenetic explanation for the most common circle of Willis variant (the "fetal" PCA) called as such when early "fetal" arrangement of PCA origin from the ICA continues to exist in the adult form, which is between $20-25 \%$ of the time. The variability in number is due to disputes over the se- mantics of what actually constitutes a "fetal" PCA. The definition is varied but it is crucial to realize that there is not such an example like an "absent" PCOM or P1. There is definitely an angiographic, MRA or CTA absence of these vessels, that means these equipment are not very sensitive to visualize it (9). The PCA can be categorized during its premesencephalic path into two segments; P1 segment that is located in inter crural cistern and P2 segment that is located in the crural, along the uncus and ambient cisterns (10).

Early development of the PCA is presented by its supply of the lateral and third ventricular choroidal territory coupled with the anterior choroidal artery. As a matter of fact, from a phylogenetic point of view, it is the anterior choroidal artery (AChA) that supplies as the artery to the occipital and temporal lobar areas, not the PCA. In the human, the anterior choroidal parenchymal artery supply is restricted to its segment proximal to its plexal point, with hemispheric territory transferred to the PCA. However, on occasion the AChA keeps some of its formerly extensive cortical possessions, and therefore might be mistaken for a fetal PCA. In the great majority of cases, however, the PCA is responsible for the supply of the mesial occipital, inferomesial parietal, and inferior temporal lobes, as well as the choroid plexus of the lateral and third ventricles (together with the AChA). Importantly, it also provides supply of the cerebral peduncles and the collicular plate which is phylogenetically considered the older territories (9).

The middle and posterior hippocampal arteries which mostly arise directly from the PCA, have a straight path on the surface and when they approach the hippocampus, they move parallel to the superfcial hippocampal sulcus and the margo denticulatus. Along their longitudinal terminal segment, the hippocampal terminal arteries phylogenically arise and end into large and small subdivisions. The large subdivisions directly penetrate the hippocampus in the deep sulci between the dentes of the margo denticulatus at a right angle which are only seen in primates. The small subdivisions penetrate the whole surface of the margo denticulatus. Some small parallel arteries (straight arteries) have a rectilinear path on the margo denticulatus and then penetrate the hippocampus (10). 


\section{Phylogeny and evolution of anterior choroidal artery}

The AChA supplies a significant portion of cortical territory until late in evolution that in the human is joined by the PCA. This connection is determined by the proximity of the two vessels in the subarachnoid space around the midbrain (11). The AChA system is composed of two parts, one belongs to the cranial division of the ICA and supplies the paleostriatum and piriform cortex, the other belongs to the caudal division and supplies the rest of the anterior choroidal territories. This is the caudal part that supplies the hippocampus. The AChA is a peculiar artery, considering its territory that supplies and its small size. Its origin is variable; it can be a branch either of the cranial division of the ICA (because of its phylogenie past) or of its caudal one (PCoA) (through its cortical link with the diencephalo-mesencephalic origins). In most cases it will give rise from the ICA anterior division (10).

\section{Intra-hippocampal (deep) arteries}

The intra-hippocampal arteries are distinguished by their twisted path following of the cornue Ammonis and the gyrus dentatus. They are branched into large ventral, large dorsal, small ventral and small dorsal intra-hippocampal arteries. The large ventral intrahippocampal arteries get into the hippocampus between the dentes of the margo denticulatus. The large ventral arteries supply CA1 and, by their terminal branches, CA2. Arterial branches arising from the large ventral arteries often have an oblique and extended course through CA1. In contrast, the large dorsal intrahippocampal arteries are shorter, twisted shape. They pass between the dentes of the gyrus dentatus and then situated inside CA4 along the gyrus dentatus. Their terminal segment reaches $\mathrm{CA} 3$, and occasionally $\mathrm{CA} 2$, through a sharp curve. Along their route, the large dorsal arteries also supply CA4 and the distal portion of the gyrus dentatus. The branches to the gyrus dentatus have a long, straight course (10).

\section{Variations and collateral anastomosis}

Anastomoses between anterior hippocampal artery (AHA), middle hippocampal artery (MHA), and posterior hippocampal artery (PHA) can be identified in a significant number of cases (12). The intrahippocampal arteries present with variations. For example, an artery originating from the large ventral or dorsal group may supply a territory extending to almost the whole hippocampus. Mostly CA1 is supplied by the large ventral arteries, whereas CA2-CA4 and the gyrus dentatus have several incoming arteries coming from different arterial groups. This specific arterial supply of CA1 and the long route of its arterial subdivisions have been in favor of a vascular theory describing the selective vulnerability of CA1 to anoxia. Against this theory it could be emphasized that the gyrus dentatus, like CA1, is supplied by fine, long arteries, whereas it is mostly believed as a subdivision which is resistant to anoxia (10).

\section{White matter, phylogeny and circulation}

The brain enlargement in humans has been followed by disproportionate enhancing in specific regions. Some alterations of internal organization may be anticipated because of functional, developmental, or constructional restrictions that need redesign with changes in total brain size. For example, in humans compared to other primates there is extra white matter underneath the neocortex. The proportion of neocortical white matter volume in humans, matches with allometric measuring expectations based on the demands for interconnections of gray matter. Additionally, the human neocortex (gray and white matter combined) holds a large fraction of total brain size than it does in great apes. While most of this additional neocortical growth could be described by evolutionarily conserved schedules of neurogenesis, it is important that the size of the human neocortex actually surpasses what would be anticipated for an anthropoid primate of the same brain size (4).

In addition to the maturational changes of major cerebral arteries, the microcirculation of the brain undergoes an important developmental evolution. The evolution affects the vulnerability of the fetus and preterm infants to lesions seen rarely after 36 weeks of gestation, such as extensive white matter infarcts, periventricular leukomalacia, and germinal matrix hemorrhages. The penetrating vessels supplying the white matter of the cerebral mantle are mostly end-arterioles with few anastomoses and little collateral circulation in the fetus and premature (13). 
Duvernoy describes six types of perforating arteries in mature intracerebral vascularization (excluding the arteries of the basal ganglia), depending on their degree of penetration into the brain: Type 1 is the most superficial and supplies only the outer, molecular layer. Type 2 reaches the pyramidal layer. Type 3 is the most abundant and supplies the medial granular layer and the closest portions of the adjacent layers. Type 4 reaches the deepest layers of the cortex. Type 5 reaches the subcortical white matter and the adjacent deep cortex and type 6 (the medullary arteries of Duret) do not supply the cortex but only the white matter lining the ventricular wall (9). Leptomeningeal arteries comprise the terminal branches of the cerebral and cerebellar arteries, which penetrate the cortex and subjacent white matter. Infarcts involving the leptomeningeal artery system are often described as territorial infarcts (11).

Clinically, understanding the evolution and development of white matter circulation is important as both the germinal matrix and periventricular white matter are border zone regions and the risk for ischemic injuries is significantly increased during periods of systemic hypotension, particularly in a pressure-passive cerebral circulation in these areas (14).

\section{Clinical implications}

Reviewing the brain circulation evolution and development is fundamentally important to understand the brain circulation diseases including stroke. For instance, small infarcts (lacunae) have often been reported in the basal ganglia or the subcortical white matter supplied by the deep perforators of the ICA (25). The collateral circulation plays a crucial role in the pathophysiology of cerebral ischemia. Intracranial collateral circulation represents a vascular network which is dynamically recruited after occlusion of cerebral arteries to provide a source of residual blood flow (26). Past researches have shown that the infarct in the territory of the deep perforators from the carotid system (ITDPCS) are most commonly involved the territory of the lenticulostriate arteries followed by the AChA and watershed zones between these two territories (27). In other words, lateral lenticulostriate territory is responsible of more than half of the infarcts that occurs in the territory of the deep perforators from the carotid system (13). White-matter hyperintensities (WMHs) may also have lacunes at the edges along the course of perforating vessels supplying the respective brain region that could be a factor in the enlargement of the WMH (28). The striatal arteries develop a muscularis wall at approximately 24 weeks' gestation but most other cerebral vessels do not form a smooth muscular wall until the last few weeks of the fetal life. The muscularization of intra-cerebral arteries occurs in a centripetal direction. The maturational changes of major cerebral arteries undergo developmental evolution that affect the vulnerability of the fetus to some lesions that would rarely be seen after 36 weeks of gestation, such as extensive white matter infarcts, periventricular leukomalacia, and germinal matrix hemorrhages. The penetrating vessels supplying the white matter are mostly end arterioles with few anastomosis and little collateral circulation in the fetus. In addition, the vascular channels of germinal matrix are immature, thin-walled vessels. Their thin endothelial cells are easily damaged by hypoxia and acidosis (29). Furthermore, cognitive disorders like dementia may also be related to focal cerebral lesions of vascular origin or chronic ischemia of the white matter due to arteriosclerosis or lipohyalinosis of small perforating arteries following high blood pressure during mid-life and after (30). Technically, there are methods like arterial pulsatility index (PI) to measure the vascular resistance of an artery, which is done by using transcranial Doppler ultrasonography (TCD) that reflects the vascular resistance distal to the artery being examined. Any pathology of small intracranial perforating arteries may affect the PI of the proximal artery (31).

\section{REFERENCES}

1. Tam S.J., Watts R.J. Connecting vascular and nervous system development: angiogenesis and the blood-brain barrier. Annu Rev Neurosci. 2010; 33:379-408.

2. Hawkins B.T., Davis T.P. The blood-brain barrier/neurovascular unit in health and disease. Pharmacol Rev. 2005; 57(2):173-85.

3. Velut S. Embryology of the cerebral veins. Neurochirurgie.1987. 33(4): 258-63.

4. Truwit C.L. Embryology of the cerebral vasculature. Neuroimaging Clin N Am, 1994. 4(4):663-89. 
5. Klosovskii B.N. Fundamental facts concerning the stages and principles of development of the brain and its response to noxious agents. In: Haigh. The development of the brain and its disturbance by harmful factors. London, Pergamon Press; 1963;3-43.

6. Raybaud C. Normal and abnormal embryology and development of the intracranial vascular system. Neurosurg Clin N Am. 2010; 21(3):399-426.

7. Martin N.A., Vinters H.V. Arteriovenous malformations. In: Carter LP. Neurovascular surgery. McGraw-Hill. 1995; 48, 875-6.

8. Streeter G.L. The developmental alterations in the vascular system of the brain of the human embryo. Contrib Embryol, 1918. 8: 5-38.

9. Lasjaunias P., Berenstein A., Ter Brugge K.G. Surgical Neuroangiography: Clinical vascular anatomy and variations. Volume 1. 2nd ed. New York: Springer- verlag Berlin-Heidelberg 2001. 480-629.

10. Lasjaunias P., Santoyo-Vazquez A. Segmental agenesis of the internal carotid artery: angiographic aspects with embryological discussion. Anat Clin. 1984; 6(2):133-41.

11. DeReuck J.L. Embryologic and anatomic bases of human brain/ spinal cord circulation and stroke. In: Ginsberg MD, Bogousslavsky J, editors. Cerebrovascular Disease: Pathophysiology, Diagnosis and Management. Malden, MA: Wiley-Blackwell; 1998; 935-8.

12. Silver J.M., Wilkins R.H. Persistent embryonic intracranial and extracranial vessels. In: Wilkins RH, Rengachary SS. Neurosurgery update II. McGraw-Hill. 1991; 59, 50-59.

13. Ghika J.A., Bogousslavsky J., Regli F. Deep perforators from the carotid system. Template of the vascular territories. Arch Neurol. 1990; 47(10):1097-100.

14. Saikia B., Handique A., Phukan P., Lynser D., Sarma A. Circle of Willis: Variant forms and their embryology using gross dissection and magnetic resonance angiography. Int J Anat Res 2014, Vol 2(2):344-53.

15. Williams P.L., Banister L.H., Berry M.N., Collins P, Dyson M., Dussek J.E., Ferguson M.W.J. Gray's Anatomy. 38 ${ }^{\text {th }}$ Edition. New York: Churchill Livingstone 1995; 295-305.

16. Davidson A.S., Stoodley M.A. Central nervous system vascular malformations. In: Sutcliffe A. Congenital Anomalies - Case Studies and Mechanisms. ISBN: 978-953-51-0075-1, InTech: www. intechopen.com

17. Komiyama M., Nakajima H., Nishikawa M., Yasui T. Middle cerebral artery variations: duplicated and accessory arteries. AJNR Am J Neuroradiol. 1998; 19(1):45-9.
18. Sörös P., Whitehead S., Spence J.D., Hachinski V. Antihypertensive treatment can prevent stroke and cognitive decline. Nat Rev Neurol. 2013; 9(3):174-8.

19. Goldberg H.I. The anterior choroidal artery. In: Newton TH, Potts PG, eds. Radiology of the Skull and Brain. St Louis, Mo: CV Mosby Co; 1974; 2:1628-1639.

20. Umansky F., Gomes F.B., Dujovny M., Diaz F.G., Ausman J.I., Mirchandani H.G., Berman S.K.J. The perforating branches of the middle cerebral artery. A microanatomical study. Neurosurg. 1985; 62(2):261-8.

21. Gumus T., Onal B., llgit E.T. Bilateral persistence of type 1 proatlantal arteries: report of a case and review of the literature. AJNR Am J Neuroradiol. 2004; 25(9):1622-4.

22. Lasjaunias P., Theron J., Moret J. The occipital artery. Neuroradiology. 1978; 15:31-37.

23. Cui Y., Xu T., Chen J., Tian H., Cao H. Anatomic variations in the anterior circulation of the circle of Willis in cadaveric human brains. Int J Clin Exp Med. 2015,15; 8(9):15005-10.

24. Makowicz G., Poniatowska R., Lusawa M. Variants of cerebral arteries - anterior circulation. Pol J Radiol. 2013; I; 78(3):42-7.

25. Fisher C.M., Curry H.B. Pure motor hemiplegia of vascular origin. Arch Neurol. 1965; 13:30- 44.

26. Liebeskind D.S. Collateral circulation. Stroke. 2003; 34(9):2279-84

27. Ghika J., Bogousslavsky J., Regli F. Infarcts in the territory of the deep perforators from the carotid system. Neurology. 1989; 39(4):507-12.

28. Duering M., Csanadi E., Gesierich B., Jouvent E., Herve D., Seiler S. et al. Incident lacunes preferentially localize to the edge of white matter hyperintensities: insights into the pathophysiology of cerebral small vessel disease. Brain 2013; 136: 2717-2726.

29. Santos C.C., Sarnat H.B., Roach E.S. Cerebrovascular disorders. In: Menkes JH, Sarnat HB, Maria BL. Child neurology. Lippincott Williams and Wilkins. 2006; 13: 847-849.

30. Hanon O. Hypertension and dementia. Ann Cardiol Angeiol. 2014; 63(3):204-8.

31. Lee K.O., Lee K.Y., Lee S.Y., Ahn C.W., Park J.S. Lacunar infarction in type 2 diabetes is associated with an elevated intracranial arterial pulsatility index. Yonsei Med J. 2007, 31; 48(5):802-6.

Conflict of interest: none declared Financial support: none declared 